Presented at the Workshop on Collective States in Nuclei, Suzhou University, China, September 8-18, 1983; and to be published in the Proceedings
L.PI --17062

DE84: 005717

\title{
NUCLEAR MOMENTS OF INERTIA AT HIGH SPINS
}

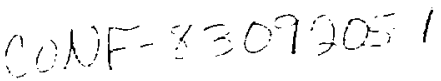

\section{A. DELEPLANQUE}

Nuclear Science Division, Lawrence Berkeley Laboratory, University of California, Berkeley, CA 94720, U.S.A.

Nuclei with highest angular monentum are discussed. The productic of high spin states, and the basic ideas associated with high spin physics sre reviewed. Recent developments from "continuum" r-ray studies are presented: the measurement of different average moments of inertia gives new information on the interplay between collective and single particle aspects at high spins. Finally, the exciting possibility of resolving the "continuum" spectra with new detecto: systems is examined.

\section{l. INTRODUCTON}

Angular momentum is a dimension along which nuclear properties can be e:amined. Just as informatior, cn deformation and shell effects came from studying a series of nuclei, the properties of the nuclei for a series of spins will show its response to new forces, namely coriolis and centrifugal, which are large. Also the highest spin studies I shall consider here separate from the more conventional "discrete y-ray spectroscopy" since we have to deal with average properties measured on "continuum y-ray spectra." This report will show recent progress made in identifying specific orbital effects and shape effects at the highest spins. It will also emphasize the exciting possibilities offered by the new generation of detectors, especially large arrays of compton..suppressed germanium detectors.

\section{PRODUCTION OF THE HIGHEST SPIN STATES}

\subsection{Spin limit}

At very high angular momentum, the nucleus tends to fission. This wili ? imit the spin values at which nuclear structure can be observed through $y$-ray decay. The liquid drop model, which reprocuces well the gross average properties of the nucleus, estimates this ":ring spin (Figure 1). The angular momentum at which the fission barria is $8 \mathrm{MeV}$ is plotted (dashed line) as a function of nucleon numbe- for nucle: along the stability line: since in the fission process, a lot of angular mentum is tied up in the relative motion of the fragments and not in the nucleus itself, particle evaporation must effectively compete with fision in order to leave high spin in the residual nuclei. This is empirically found to happen for a fission barrier 
greater than $8 \mathrm{MeV}$. For the heavier nuclei, neutrons are most of ten emitted, and they carry very little angular momentum. For the lighter nuclei, where the Coulomb barrier decreases, a particles are preferentially emitted and they can carry a lot of angular momertum, limiting further the highest spin attainable ${ }^{2}$ (dotted line). Thus, the maximum spins produr $\mathrm{d}$ are around $70 \mathrm{~h}$ and are located in the rare earth region $(A \sim 150)$. This is the region $I$ 'll discuss here.

\subsection{Production of high spin states}

A compound nucleus is formed at high excitation energy ( $\sim 80 \mathrm{MeV})$ and the angular momentum distribution $\sigma(I)$ for the evaporation residues is roughly triangular ( $\sigma$ proportional to $2 I+1$ ) up to the maximum spin. The excitation energy is most often carried away by a few neutrons down to one neutron binding energy ( $-8 \mathrm{MeV})$ above the yrast line. Below that level, called the "entry limit," $\gamma$-ray emission is predominant. This is the region of nuclear structure studies, as shown in Figure 2, on an excitation energy versus spin plot. Two kinds of $r$-rays deexcite the nucleus: i) the "statistical" $y$-rays ${ }^{3}$
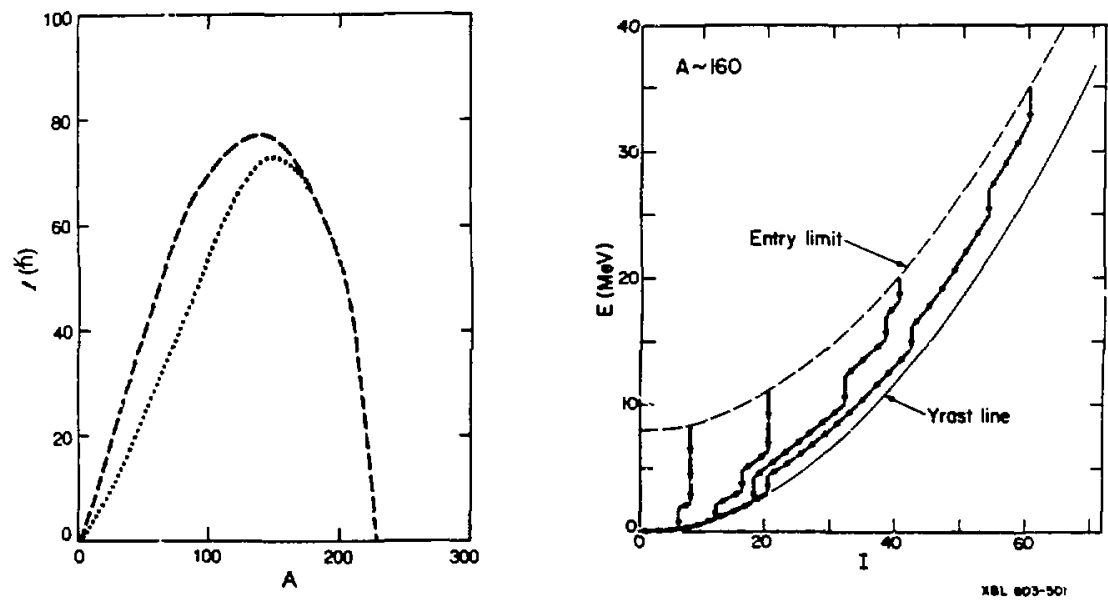

FIGURE 1

Rotating liquid drop calculation of the angular momentum at which the fission barrier is $8 \mathrm{MeV}$ (dashed line). The dotted line is an experimental estimate of the angular momentum below which FIGURE 2

Typical $\gamma$-ray deexcitation patis. vertical arrows indicate statistical transitions. Arrows parallel to the yrast line represent yrast-like transitions. 
remove the energy (vertical arrows). They are fast and their lifetime is approximately independent of spin. ii) The "yrast-like" r-rays remove the angular momentum (arrows parallel to the yrast line). Most often, and in particular in rotational nuclei considered here, they are the collective stretched E2 transitions. Their energy $E_{Y}$ increases with spin, and their transition probability, proportional to $E_{Y}^{5}$, increases with spin. At low spin they are slower than the statistical transitions, so that the population is concentrated on the yrast line. But above spin around $40 \mathrm{~h}$ the two kinds of transitions compete, which results in many different pachs for the nucleus. Individual lines are no longer separ,ble and we detect an "unresolved" or "continuum" r-ray spectrum.

\subsection{Detection of high spin states}

Those two kinds of - ray are separable on a continuum $r$-ray spectrum. Figure 3 shows such a spectrum obtained from a $(12.7 \times 15 \mathrm{~cm})$ NaI scintillator, which has a poor resolution but a rather good peak to total ratio $(-50 \%)$, so that the spectrum can be reasonably well unfolded. From its exponential tail, a "statistical" spectrum can be subtracted (dashed line). The remaining "yrast" part of the spectrum contains most of the nuclear structure information.

\section{BASIC IDEAS SPECIFIC TO HIGH SPINS}

The nucleus is a many-body system and therefore will have some macroscopic "classical" properties. But it is also a quantal system of finite size, so that the classical properties will be modified by quantal effects which sometimes single out some particular orbital.

\subsection{Nuclear shapes}

\subsubsection{Classical shapes}

The low-lying excitations correspond to the rotational degree of freedom. we shall neglect the pairing correlations which are expected to be quenched at high spins. Classically, the most favored shape will be that which minimizes the rotational energy of a rigid body, or maximizes its moment of inertia. If an axially symmetric nucleus is considered, for example, the shape is characterized by the parameter $B=\Delta R / R$, the difference in radius along 2 axes divided by the average radius. The optimum conditions correspond to an oblate (disc-like) nucleus rigidly rotating around its symmetry axis (OS). The next lowest energy is that of a prolate (cigar-like) nucleus rotating around an axis perpendicular to the symetry axis $\left(P_{1}\right)$. The other two possibilities are $0_{1}$ and PS. 


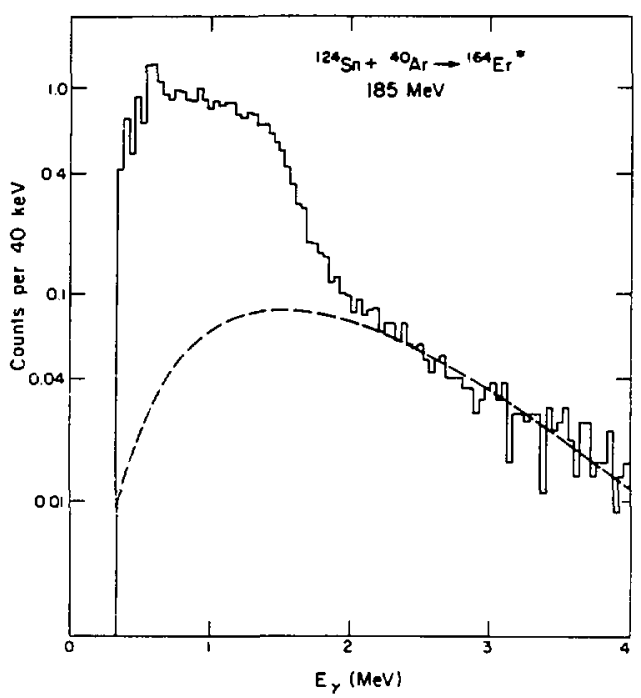

$x+1001279$

FIGURE 3

Isotropic r-ray spectrum (histogram), normalized

to the r-ray multiplicity, from the deexcitation

of high spin states of the compound nucleus $164 \mathrm{Er}$.

Its statistical part is shown as a dashed line.

\subsubsection{Quantal effects}

As a quantal system, the nucleus has a shell structure, which in turn depends on its shape. At low spin, those "shell effects" and the pairing correlations can reverse the classical order of shapes. At high spins, the shell effects ${ }^{4}$ (of order 3-4 MpV) are small enough that the two highest, trajectories $\left(O_{1}\right.$ and $\left.P S\right)$ can be ignored. They can, however, change the order of the lowest two (OS and $P_{1}$ ). More exact calculations (like the cranking model) a?so give the same results. In aodition, they also show that nuclei near major shells tend to be oblate or spherical, nuclei near the middle of a shell tend to be prolate. The classical picture is also perturbed by another property of a quantal object: it cannot rotate around its symretry axis. But Bohr and Mottelson have shown ${ }^{5}$ that, on average, the energy of such a system is the same as if it were rigidly rotating around that axis, so that the conclusion drawn above is still valid. For that reason, the behavior of suck: a system is called an "effective" rotation around its symetry axis. But the characteristics of its $\gamma$-ray deexcitation are very different from those of a good rotor: the transitions are non-collective, irregular and can be of 
dipole or quadrupole type. These properties are just a result $r_{i}$ the fact that such a motion is contained in the single-particle degrees oi freedom. For a nucleus rotating around an axis perpendicular to the symmetry axis, the rotation is collective and gives rise to regular "rotational" bands of collective stretched $E 2$ transitions $E_{\gamma}$. They define the "rotational frequency" $\omega$ which is approximately equal to $E_{\gamma} / 2$. The frequency cannot be measured experimentally for a non-collective nucleus, but the notion of effective rotation is behind the extension of the cranking model to the non-rotating nuclei. The frequency is then a parameter: the nuclear potential is rotated with a frequency w either around the symmetry axis or around an axis perpendicular to the symmetry axis. Properties of the nucleus--total energy, spin--can then be calculated as a function of frequency, and in most cases can be compared with experiment (when $w$ can be measured). The rotational frequency is presently considered as a new dimension along which many nuclear properties calculated and measured.

3.2. Interplay between collective and single particle effects

Even rotational nuclei do not rotate like a rigid body. This happens because the Coriclis force is strongest for the orbitals of highest angular momentum $j$. The coriolis force tends to align the particle angular momentum $j$ on the rotation axis, thereby decoupling its motion from that of the rest of the nucleus. The collectivity therefore decreases. This is the first step towards the non-collective situation described above. All intermediate situations are possitle and characterized by the parameter $\gamma$ which varies from $0^{\circ}$ for the collective case $\left(P_{1}\right)$ to $60^{\circ}$ for the non-collective behavior (OS).

In general there are two kinds of contributions to the angular momertum as shown on Figure 4 for ${ }^{158} \mathrm{Er}$. The smooth regular increase in spin comes from the collective motion. The sharp increase at $\hbar_{\omega} \approx 0.28 \mathrm{MeV}$ and in the spin region 10-16 is due to the alignment of a pair of neutrons (in this case $\checkmark i(3 / 2)$ on the rotation axis. This is the single-particle contribution to the angular momentum. The region of spin between $16^{+}$and $24^{+}$thus represents a collective band constructed on a $\left(v{ }^{i} 13 / 2\right)$ two-quasiparticle states. The change $i$ in the nuclear alignment at that crossing is approximately the difference between the spins in ihe two "unperturbed" bands at a given $f$ enuency.

The frequency of the crossing is characteristic of the orbitals involved. In a cranking model diagram, which shows single perticle energies in the rotating frame as a function of frequency, this corresponds to the crossing of two orbitals: two-quasiparticle srbitals at low spins, or two single-particle 
$-6-$

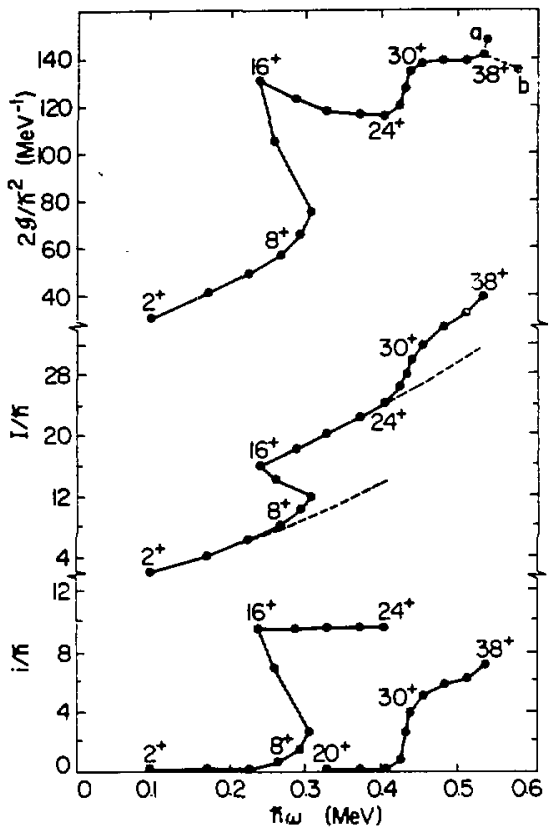

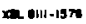

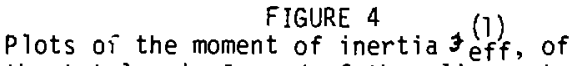
the total spin $I$, and of the alignment $i$ as a function of frequency for the nucleus $158 \mathrm{Er}$.

orbitals at high spins where the pairing correlations are neglected. Figure 5 gives such a diagram for protons around mass 165 . The alignment is the slape of those orbitals. Near $z=66$, there are only a few downsloping (aligned) orbitals, mainly $541 \mathrm{l} / 2\left(\mathrm{~h}_{9 / 2}\right)$ and $6601 / 2\left(i_{13 / 2}\right)$, and they reach the Fermi level at a frequency close to $0.6 \mathrm{MeV}$. In that $\mathrm{m}$. $s$ s region this frequency corresponds to a spin around $45 \mathrm{f}$, i.e., in the region where the $r$-ray spectra are unresolved and many bands deexcite the nucleus. Schematically, one expects that those aligned orbitals become populated in essentially all the bands around $\hbar_{\omega}=0.6 \mathrm{MeV}$. But since all these bands would differ by the rest of the configuration, it is not clear whether these many crossing frequencies would be similar enough to permit identification of the aligning orbital. This is still an open question.

3.3. Collective and effective moments of ineria

Since we cannot resolve individual bands at high spins, we cannot identify 
band crossings as shown in Figure 4 . This question must be addressed differently, through the notion of moments of inertia.

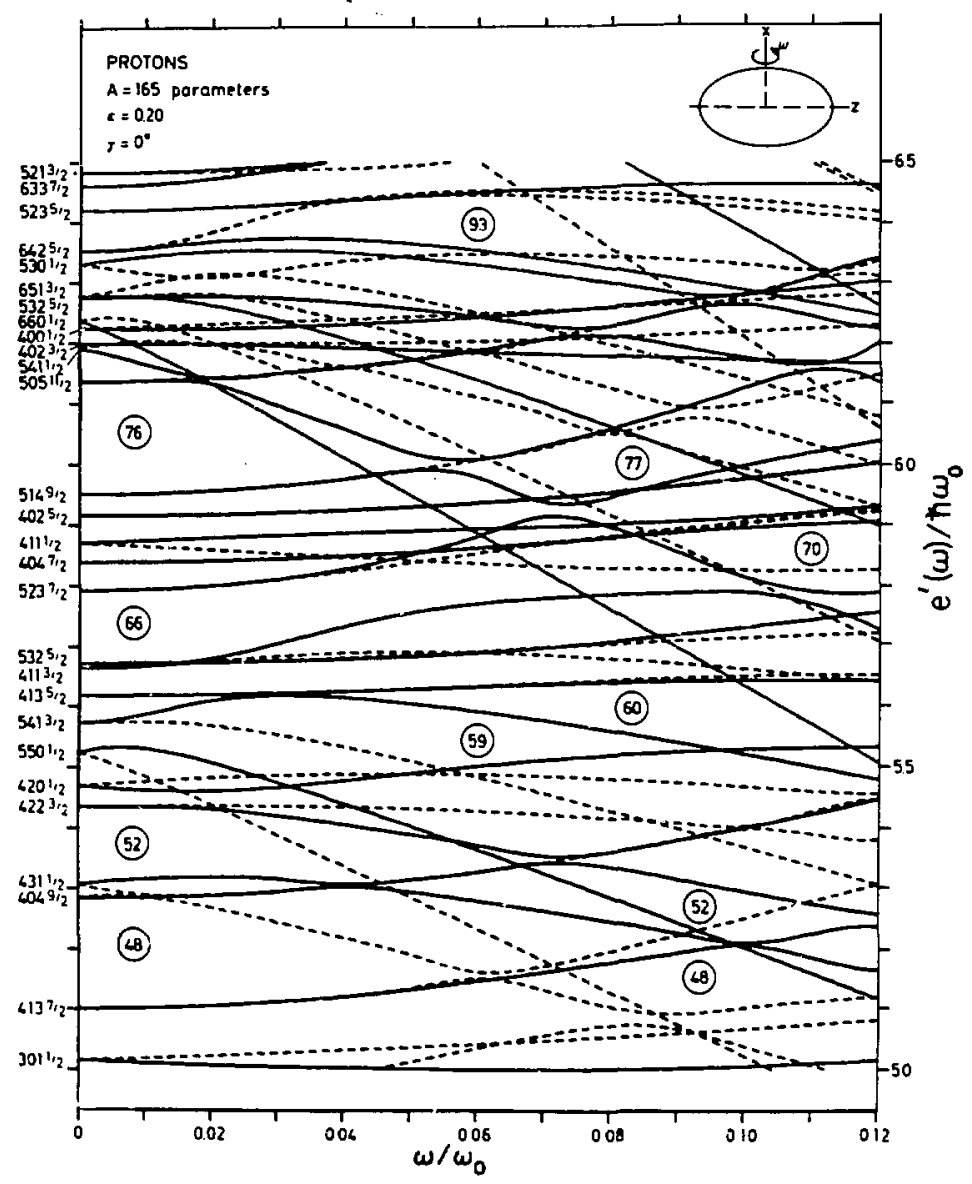

XBL 806-988B

\section{FIGURE 5}

Example of energy levels as a function of rotational frequency (in units of oscillator frequency $\omega_{0}$ ) calculated with a cranked modified oscillator potential (from Ref. 7). The solid and dashed lines correspond to states of different signatures.

They are of two kinds: the kinematic moments of inertia, which are related to the overall motion of the nucleus, and the dyramic moments of inertia, which 
describe its response to the torque. For a perfect rotor with a moment of inertia $\}$, the rotational energy at spin $I$ is $E \approx \hbar^{2} I^{2} / 2 \mathcal{F}$. Its first derivative with respect to spin: $d E / d I=\hbar^{2} I / \mathcal{F}=E_{Y} / 2=\hbar_{\omega}$, is related to the $k$ inematic moment of inertia $\xi^{(1)}$ defined as $z^{\gamma}(1) / \hbar^{2}=I(d E / d I)^{-1}=I / \hbar_{\omega}$. The second derivative $d^{2} E / d I^{2}=\hbar^{2} / \xi=\hbar d \omega / d I$ is related to a dynamic moment of inertia $\mathcal{f}^{(2)}: z^{(2)} / \hbar^{2}=\left(d^{2} E / d I^{2}\right)^{-1}=d I / \hbar d \omega . y^{(2)}$ represents the rate of change in spin with frequency. Those two moments of inertia are identical for a perfect rotor. But when there are changes in the internal structure of the nucleus, such as alignment of single-particle angular momentum, 1/w will be different from $d I / d_{\omega}$ reflecting the fact that the nuclear motion is not the simple rotation of a rigid body. The moment of inertia $f^{(1)}$ at a frequency $\omega$ is an average of $y^{(2)}$ over a frequency region from 0 to $\omega$ and therefore will be much less sensitive to variations with frequency. We will therefore concentrate on the determination of dynamic moments of inertia $f^{(2)}$.

Those moments of inertia can be defined for any sequence of levels, but two of them play an important role in the decay modes of the nucleus. The characteristic feature of collective motion is the rotational band (see figure 4). It is therefore natural to define a moment of inertia $\mathcal{f}_{\text {band }}^{(2)}=(d I / d \omega)$ band by calculating $d I / d_{\omega}$ only in the band region. Provided the alignment and the deformation are reasonably constant within a band, $\mathcal{F}_{\text {band }}^{(2)}$ is related to the collectivity of the motion. If $d I / d \omega$ is considered for a whole decay path, both co lectivity and alignment will be taken into account and therefore an effective moment of inertia is defined by $\mathcal{F}_{e f f}^{(2)}=(d I / d \omega)$ path. For a single decay path $\mathcal{f}_{\mathrm{eff}}^{(2)}=\mathcal{F}_{\text {band }}^{(2)}$ in the band regions, but $\mathcal{f}_{\mathrm{eff}}^{(2)}$ can become very large in the regions of alignment (band crossing?.

Both $f_{b \text { and }}(2)$ and $f_{\text {eff }}^{(2)}$ can be calculated as a function of frequency in the cranking model and measured experimentally. However the measured values represent an average over many decay paths and therefore will probabiv be smeared as compared to the theory. Nevertheless, the comparisons should be very useful since they are at present the only contact between theory and experiment.

๑. EXPERIMENTAL DETERMINATION OF MOMENTS OF INERTIA AT HIGH SPINS

4.1. Selection of high spin states

Because the $\gamma$-rays are emitted in a i imited domain (extending from 0 to -8 MeV, see Figure 2) above the yrast line, the total r-ray energy is related to the initial spin. Therefore high spin states are selected by a high total 
r-ray energy $E_{\text {tot }}$. $E_{\text {tot }}$ is measured in a high efficiency device: it can be two large $\mathrm{NaI}$ scintillators, or a "ball" composed of many scintillators. In coincidence with those, the $\gamma-r a y$ spectra are obtaired in a number of $\gamma$-ray detectors, placed at different angles. They are far away from the target to avoid pile-up effects and to discriminate against reutrons by time of flight.

4.2. Determination of $\mathcal{F}_{\text {band }}^{(2)}$

The rotational band structure is easily racognizable on a $\gamma-\gamma$ coincidence plot (Figure 6). It shows a "valley" along the main diagonal (no two r-rays have the same energy) and a "ridge" structure. The width of the valley is just $4 \mathrm{dE}_{2} / \mathrm{dI}=8 \mathrm{dw} / \mathrm{dI}=16 \hbar^{2} / 2 \mathcal{f}_{\text {band }}^{(2)}$. We measure $\mathcal{F}_{\text {band }}^{(2)}$ because only in-band transitions will give rise to that regular structure. If riany bands have the same moment of inertia, the intensity will appear on the same

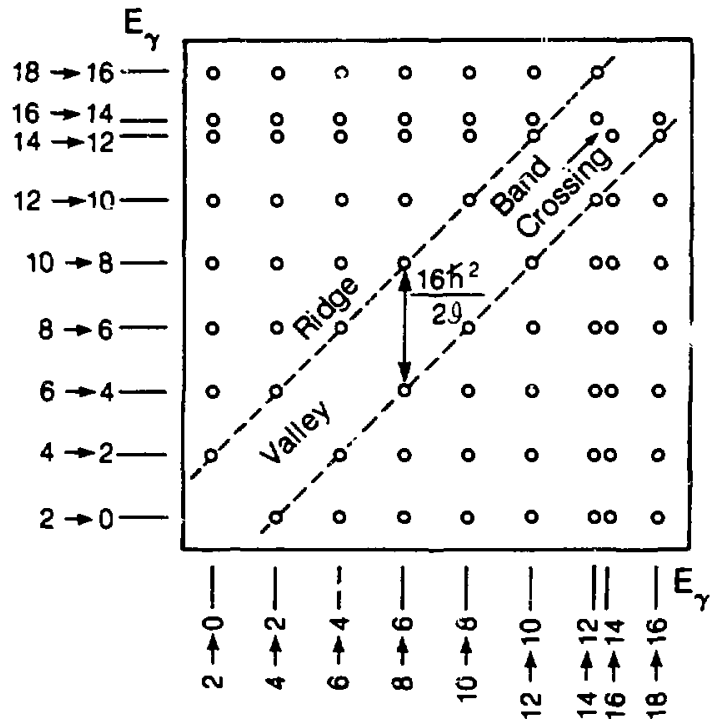

$X \theta L 6311-644$ 
ridges. Therefore, even without resolving the bands, their moment of inertia can be measured. If there were a band structure at high spins, but with a variety of moments of inertia values, the ridges would be smeared out but the valley should still be visible. The band crossings tend to fill the valley at the crossing frequency (see Figure 6). But if the crossing freruencies in each band are sufficiently similar, they should show up as a distinct peak in the valley.

The results of such measurements are still tentative, bacause of experimental difficulties to obtain good statistics. Trie poor resolution of NaI scintillators ( $50 \mathrm{KeV}$ at $1 \mathrm{MeV}$ ) tends to smear out the ridges too much so that the valley width is not well defined (it is $\sim 100$ iev in the rare-earth region). Germanium detectors however have a bad peak/tntal ratio ( $15 \%)$ so that about 98; of all events has to be removed as background. Great progress will surely be made when large arrays of Compton-suppressed germanium detectors are operational. At present the results are somewhat contradictary. In some rare earth nucle $i^{6}, \mathcal{F}_{\text {band }}^{(2)}$ seems to decrease at high frequency, as would be expected if additional alignments occur at high spins, and as some calculations seem to indicate. However, data from Darestury on ${ }^{7}{ }^{7} \mathrm{Ce}$ (the first obtained with six Compton-suppressed Ge detectors) do not indicate a decrease of $f_{\text {band }}(2)$ at high frequencies.

4.3. Determination of $\boldsymbol{f}_{\mathrm{eff}}^{(2)}$

A new--more quantitative--viewpoint on continuum $r-r$ ay spectra was reached recently when it was recognized ${ }^{8}$ that the height $d N$ of a continuum $r-r a y$ spectrum (normalized to the r-ray multiplicity) is proportiona? to $\mathcal{F}_{\text {eff }}^{(2)}$ for a spectrum composed of collective stretched $E 2$ transitions. If $d E_{r}$ is the channel width, the quantity $d N / d E_{Y}=d I / 4 d w$. Since there is no selection of in-band transitions in that spectrum, this is just $y_{\mathrm{eff}}^{(2)} / 4$. $\boldsymbol{y}_{\mathrm{eff}}^{(2)}$ describes how efficientiy the angular momentum is generated at a given frequency. The most efficient way is probably the alignment, which occurs because it is a cheap way for the nucleus to generate angular momentum. It appears as a peak in a $y$-ray (or $\xi_{\text {eff }}^{2}$ ) spectrum, since several iransitions are closer in energy.

There is an experimental probiem in determining $f_{\text {eff }}^{(2)}$ at high frequencies. These frequencies correspond to the highest spin states which, in a given total energy gate, are only partially fed. The neight of tive r-ray spectrum in that region is then $7_{\text {eif }}(2)$ times the fraction of the papulation going through trat spin (or frequency) region. We have devised a method to correct for that incomplete feeding for quasirotational spectra. 
This method is most easily understood for a perfect rotor $\left(7_{\mathrm{eff}}^{(2)}(\omega)\right.$ constant). The corresponding $r$-ray spectrum would drop at high frequency because of the incomplete feeding at high spins, and the feeding (direct population) would just be its derivative. In practice, we obtain the feeding ly taking a finite difference between two spectra corresponding to two slightly different populations selected by two slightly different total $\gamma$-energy slices.

Figure 7 shows an example of the results obtained for several Erbium nuclei. $160 \mathrm{Er}$ is a rather good rotor showing a rather flat spectrum, except for the known first ( $\hbar_{\omega}-0.28 \mathrm{MeV}$ ), blocked and second ( $\hbar_{\omega} \sim 0.45 \mathrm{MeV}$ ) backbends, and a rise at the highest ( $\hbar_{\omega}>0.6 \mathrm{MeV}$ ) frequencies. This rise is accompanied by a decrease in $f_{b \text { and }}^{(2)}$ (measured from a $\gamma-\gamma$ correlation experiment), which
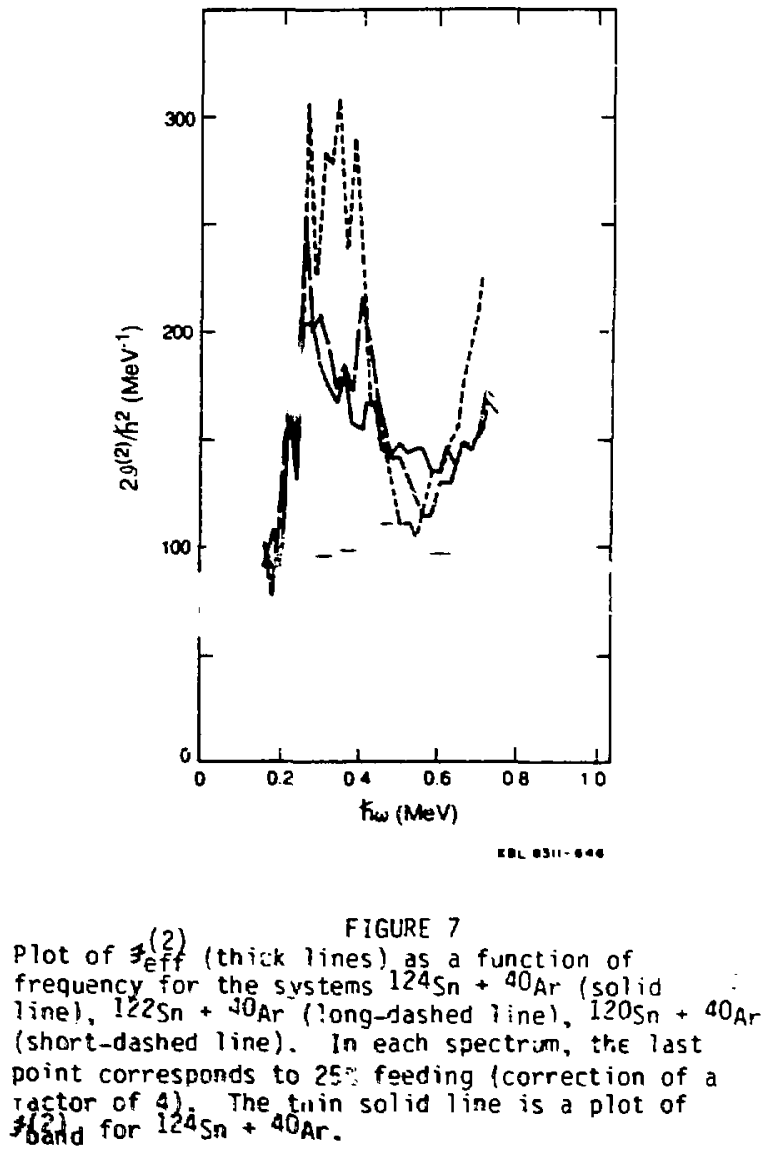
suggests it is due to alignment effects. The comparison with the $\mathcal{f}_{\text {eff }}^{(2)}$ spectrum of its isotone ${ }^{162}$ yb flirther suggests ${ }^{8}$ that this is a proton alignment of $\hbar_{9 / 2}, i_{13 / 2}$ orbitals which come down to the Fermi level (see Figure 5) around $h_{\omega} \sim 0.6 \mathrm{MeV}$ for Er nuclei.

The $f_{\text {eff }}^{(2)}$ spectra for $158,156 \mathrm{Er}$ illustrate Fermi level and shape effects. As the neutron Fermi level goes down in that region it comes cioser to the righly aligned $\Omega=1 / 2$ component; of the intruder $i_{13 / 2}$ neutron :ubshell, and the nucleus becomes less defarmed. Both these reasons make the valence shell backbends ( I Je-backbends) easier. This is what we see in the lower frequency region: in the lighter nuclei, the valence backbends are campresses in frequency which makes the lower peak taller and narrower than in the heavier nuclei. At higher frequencies, where particle alignments are expected, the Fermi level effect just described would be opposite ii.e., alignments would be delayed) for particle type backbend (like ${ }, 2,{ }_{1}, 13 / 2$ proton backbends) coming from the next shell. Although the proton Fermi level does not change from ${ }^{160} \mathrm{Er}$ to ${ }^{156} \mathrm{Er}$ the latter is softer and might generate high spins more efficiently by acquiring large deformations. This would also produce high values of $\mathcal{f}_{\mathrm{eff}}^{(2)}$. We think this deformation effect might be höppening in ${ }^{156} \mathrm{Er}$.

\section{FUTURE PROSPECTS}

Although continuum $\gamma$-ray studies begin to reveal rather fine details like single-particle effects, a much better understanding of nuclear structure at high spins would be achieved if those spectra could be resolved. With recent progress in technology, there is indeed a hope to resolve several bands up to spin around $50 \mathrm{~h}$.

The idea is to build large ariays of Compton-suppressed Ge detectors. The Compton suppression improves greatly the peak to background ratio, the more gain the higher the coincidence order. Large arrays produce high rates and therefore open the possibility of recording higher order coincidences. Multiple coincidences increase the number of "resolvable points:" for example, if there are $\mathrm{N}$ resolvable points in a given enargy interval of a single spectrum, there wi?l be $\mathrm{N}^{3}$ such points in a triple coincidence spectrim. Also triple coincidences can be used to make double gaces, with which more bunds can be separated, since each band is now characterized by two tranitions instead of one. 
In Berkeley, we are building an array of 21 Compton-suppressed Ge detectors. They are placed on three rings around a target and the central one is shown in Figure 8. This was possible because of the small size $(-12.5 \times 12.5 \mathrm{~cm})$ of the suppressors which are made of Bismuth Germanate, a scintillator material, twice as dense as sodium iodide, and recently available in large sizes. With such a system, the peak to total ratio (counted from $300 \mathrm{KeV}$ up) for a $1 \mathrm{MeV}$ r-ray is about $50 \%$. We estimate that using triple concidences we will be able to separate ten times miore bands than we can with only double coincidences.

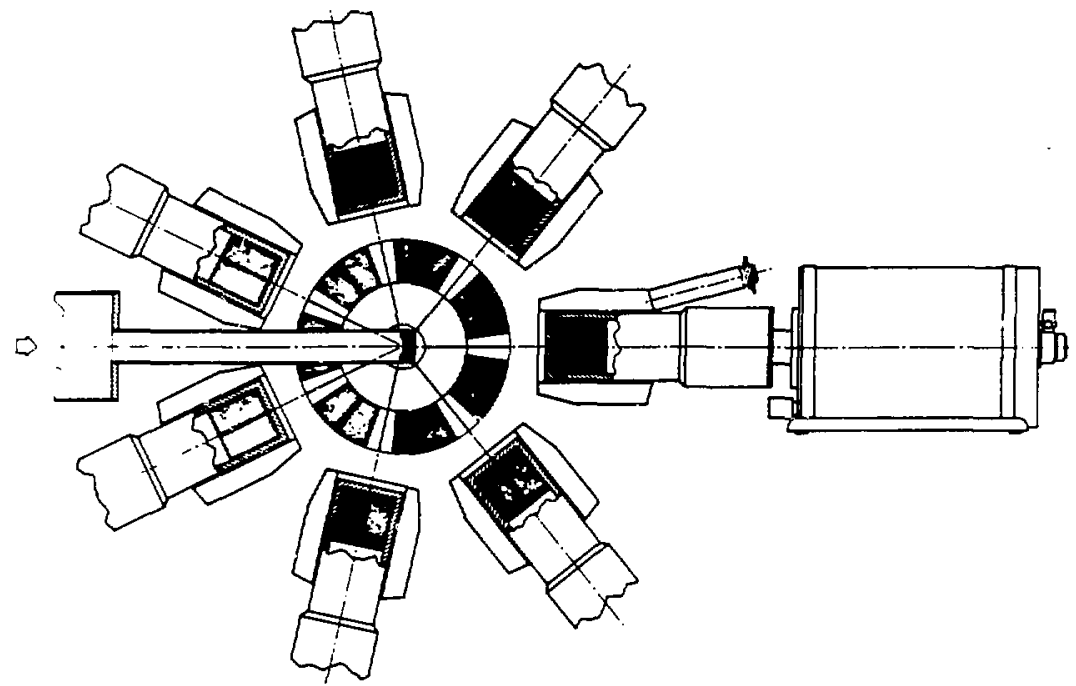

SBL $819-2053$

FIGURE 8

Cross-section of the central ring of seven Compton-suppressed germanium detectors. The beam comes from the left and the target is in the center. Also shown is the cross-section of a central Bismuth-germanate ball.

\section{CONCLUSION}

Continuim r-ray studies have considerably enriched our knowledge of high spin properties of the nucleus. They lead to new concepts like the existence of several moments of inertia and their relation to single particle and collective effects. The results suggest that single particle effects may be identified at the highest spins. But only qualitative trends can be measured with such methods. More quantitative spectroscopy will require resolving the spectra, which we hope to accemplish up to spin around $50 \mathrm{~h}$ in the near future. 
The results already obtained in Daresbury with the first Compton-suppressed array show exciting possibilitiès.

\section{ACKNOWLEDGEMENT}

This work has been done in collaboration with colleagues and visitors. I would like to thank them and especially R.M. Diamond and F.S. Stephens. This work was supported by the Director, Office of Energy Research, Division of Nuclear Physics of the Office of High Energy and Nuclear Physics of the U.S. Depa tment of Energy under Contract No. DE-ACO3-76SF00098.

\section{REFERENCES}

1) S. Cohen, F. Plasil and W.J. Swiatecki, Ann. Phys. 82 (1974) 557.

2) J.0. Newton et al., Phys. Rev. Lett, 38 (1977) 810.

3) R.M. Diamond and F.S. Stephens, Ann. Rev. Nucl. Part. Sci. 30 (1980) 85.

4) G. Andersson et al., Nucl. Phys. A268 (1976) 205.

5) A. Bohr and B.R. Mottelson, Nuclear Structure, Vol. 2 (W.A. Benjamin, Reading, 1975).

6) M.A. Deleplanque et al., Phys. Rev. Lett. 45 (1980) 172.

7) P.J. Nolan, Phys. Lett. 128B (1983) 285.

8) M.A. Deleplanque et al., Phys. Rev. Lett. 50 (1983) 409. 
This report was done with support from the Department of Energy. Any conelusions or opinions expressed in this repor represent solely those of the author(s) and not necessarily those of The Regents of the University of California, the Lawrence Berketey Laboralory or the Department of Energy.

Reference to a company or product name does not imply approval or recommendation of the product by the University of California or the U.S. Deparment of Energy to the exelusion of others tliat may be suitable. 